\title{
La asistencia sanitaria en tiempos de epidemia en Río de Janeiro en el siglo XIX
}

\author{
Tânia Salgado Pimenta (*) \\ $\left({ }^{*}\right) \quad$ Casa de Oswaldo Cruz/Fiocruz - Brasil. taniaspimenta@fiocruz.br
}

Dynamis

[0211-9536] 2011; $31(1): 21-39$
Fecha de recepción: 2 de febrero de 2010

Fecha de aceptación: 18 de diciembre de 2010

SUMARIO: 1.-A la espera del cólera. 2.-El hospital de la Santa Casa y la atención sanitaria en Río de Janeiro en el siglo XIX. 3.- La creciente demanda asistencial en el Hospital de la Santa Casa. 4.-En tiempos de epidemias. 5.-Consideraciones finales.

RESUMEN: El objetivo de este artículo es examinar cómo se llevó a cabo, a mediados del siglo XIX, la atención sanitaria a la población de Río de Janeiro y en qué medida esta oferta fue reorganizada ante la crisis que se instaló con el estallido de las epidemias de fiebre amarilla y de cólera. Para ello, resulta indispensable considerar que la asistencia sanitaria, incluso el propio espacio donde se daba la asistencia hospitalaria, estaba experimentando cambios significativos durante este período. En este sentido, profundizamos la investigación en el hospital Santa Casa de la Misericordia, el más importante de la capital del Imperio del Brasil. Las fuentes utilizadas son la correspondencia entre el gobierno y la Santa Casa de la Misericordia, los informes anuales de esta última, los oficios de la Junta Central de Higiene Pública y las revistas médicas de amplia circulación.

Palabras clave: Historia de los hospitales, epidemia, Santa Casa de la Misericordia, historia de la salud pública, historia de Brasil.

Key words: History of hospital, epidemic, Santa Casa de Misericórdia, history of public health, history of Brazil.

(*) Este artículo es el resultado del proyecto de investigación desarrollado con ayuda de la Casa de Oswaldo Cruz y de la «Fundación Carlos Chagas Filho de Amparo à Pesquisa do Estado do Rio de Janeiro». 


\section{A la espera del cólera}

Desde la década de 1830, cuando la epidemia de cólera se extendió por Europa occidental, los médicos brasileños se impacientaban ante la llegada de esta enfermedad. Desde principios de 1831 hasta 1833, las notas sobre la propagación de la enfermedad eran una presencia constante en las revistas médicas especializadas. En febrero de 1831, la epidemia se había presentado con «toda su furia» en varias provincias de Rusia; en agosto, había llegado a Polonia y Hamburgo, y al año siguiente, a París ${ }^{1}$. A petición de la Cámara de Diputados, los médicos de la Sociedad Médica de Río de Janeiro ${ }^{2}$ presentaron algunos "consejos a las autoridades en caso de amenaza o invasión del cólera-morbus». Como había datos a favor del carácter contagioso de la enfermedad y datos a favor de su diseminación por miasmas, los miembros de la Sociedad Médica consideraron necesarias medidas como la cuarentena de los buques y la retirada de gran parte de los esclavos y «gente ociosa y miserable» de la ciudad. En septiembre, sin embargo, parecían enterradas las últimas esperanzas de que Brasil quedara fuera del camino del cólera. La enfermedad había cruzado el océano, causando sus estragos en América del Norte ${ }^{3}$. No obstante, el tempo pasó y el cólera no llegó.

Ya a finales de la década de 1840, la atención de los médicos se dirigió a la nueva expansión del cólera en varias ciudades de Europa y América del Norte. Desde los últimos meses de 1852, en casi todas las sesiones de la Junta Central de Higiene Pública ${ }^{4}$ había por lo menos una nota sobre el progreso de la enfermedad. A mediados de octubre, el Ministro del Imperio pedía a la Junta que indicase brevemente las medidas para «prevenirse de la entrada de ese flagelo.» El doctor Pereira Rego, miembro de la Junta, fue el encargado de presentar tales medidas. Desde la epidemia de fiebre amarilla, los licenciados se dieron cuenta de que ya no podían «confiar todo, como

1. Semanário de Saúde Pública (de aquí en adelante SSP), 5 Feb y 27 de Ago de 1831; 14 Jul de 1832.

2. Principal asociación de médicos en esta época, fundada en 1829 y rebautizada en 1835 con el nombre de Academia Imperial de Medicina.

3. SSP, 21 de Jul, 18 de Ago y 15 de Sep de 1832

4. Órgano creado en 1850 en el contexto de la epidemia de fiebre amarilla, pasó a centralizar la normatización sobre salud pública. Ver los trabajos de, por ejemplo, Benchimol, Jaime. Dos micróbios aos mosquitos: febre amarela e revolução pasteuriana no Brasil. Rio de Janeiro: Editora Fiocruz e Editora UFRJ; 1999. Pimenta, Tânia. Doses infinitesimais contra a epidemia de cólera em 1855. In: Nascimento, Dilene; Carvalho, Diana, orgs. Uma história brasileira das doenças. Brasilia: Paralelo 15; 2003, p. 31-51. 
antes, a la salubridad de nuestro clima». En el proyecto de ley enviado al gobierno, la Junta se mostraba consciente de que sus consejos provocarían un «choque de ciertos intereses» y serían acusados de innecesarios e inútiles. Además, consideraba que no había certeza acerca de la diseminación de la enfermedad por contagio (a través de personas o de objetos contaminados) o por infección (a través de miasmas), por eso la junta defendía que era preferible tener exceso de cautela y al mismo tiempo aplicar las medidas que evitasen el contagio, como cuarentenas, y la infección, como limpieza de basura de las calles ${ }^{5}$.

Explicadas detalladamente, las medidas propuestas se dividían en preventivas y «adoptables» en el caso de que la epidemia llegase al país. Entre las primeras, las cuarentenas constituían el punto más controvertido. La Junta de Higiene, sin embargo, se basaba en el acuerdo de salud firmado en febrero de 1852 por doce "potencias europeas», que a pesar de reconocer la ineficacia de las cuarentenas y cordones sanitarios en las dos últimas epidemias que habían asolado Europa, juzgaba indispensable su empleo. Los debates en el exterior influenciaban las discusiones en Brasil acerca de la contagiosidad o no de la enfermedad y los intentos de detener su avance y las recomendaciones para el tratamiento ${ }^{6}$.

Durante los doce meses siguientes, los movimientos del cólera fueron seguidos de cerca por la Junta Central de Higiene Pública y por la Comisión Sanitaria del Puerto de Río de Janeiro. Ambas fueron presididas por el doctor Paula Cândido y seguían de cerca la entrada de los buques: los que venían de las zonas atacadas por el cólera eran sometidos invariablemente a la cuarentena ${ }^{7}$. Las autoridades médicas prepararon para el gobierno

5. Ofícios de 13 de Oct de 1852 y 8 de Nov de 1852. Arquivo Nacional - Rio de Janeiro (de aquí en adelante AN), Ministério do Império - Junta Central de Higiene Pública (oficios y documentos diversos), paquete IS 422.

6. Kiple, Kenneth. Cholera and race in the Caribbean. Journal of Latin American Studies. 1985; 17: 157-177. Briggs, Asa. Cholera and society in the nineteenth century. Past \& Present. 1961; 19: 76-96. Arnold, David. Cholera and colonialism in British India. Past \& Present. 1986; 113: 118-151. Evans, Richard. Epidemics and revolutions: cholera in nineteenth-century Europe. Past \& Present. 1988; 120: 123-146.

7. La Comisión fue creada, por un decreto, el 3 de enero de 1853 y comenzó a funcionar el 4 de marzo del mismo año. Sobre cuarentena, tratan las correspondencias de 24 de agosto de 1853, localizada en: AN, Ministerio del Imperio - Junta Central de Higiene Pública (oficios y documentos diversos), paquete IS 4 22; 25 de octubre de 1853, localizada en: Arquivo Geral da Cidade do Rio de Janeiro (de aquí en adelante AGCRJ), Junta de Higiene, Atas, 8-2-12. 
una serie de recomendaciones durante la década de $1850 \mathrm{y}$, finalmente, a mediados de 1855, tuvieron que ponerlas en práctica.

La forma como la sociedad enfrentó la primera epidemia de cólera se basó, en gran parte, en los informes sobre la pandemia de la década de 1830 que proporcionó información a los médicos y a la prensa, alimentando los debates académicos y el temor de la población sobre la terrible enfermedad. Del mismo modo, la experiencia de la epidemia de fiebre amarilla de 18491850 acabó influenciando las reacciones frente al avance del flagelo en 1855.

Las primeras conversaciones e identificaciones de casos se dieron de manera confidencial entre las autoridades gubernamentales, la administración del hospital de la Santa $\mathrm{Casa}^{8}$ y las autoridades médicas, a fin de tratar de posponer los problemas que serían causados con el anuncio de la epidemia como el desabastecimiento de víveres y el pánico entre la población. Después de llegar a Belem, al norte de Brasil, en un buque procedente de Portugal, la epidemia se propagó por los centros urbanos, así como también vía embarcaciones ${ }^{9}$.

Al final de la tarde del día 12 de julio de 1855, fue internado en el hospital de la Santa Casa «un individuo acometido de una enfermedad». Enterada de este hecho, la Secretaría de Policía de la Corte ordenó al administrador del hospital que informase, con urgencia y detalles, del estado y la identidad de aquella persona, que terminó muriendo ${ }^{10}$. Se supo que era un «negro», que había estado en contacto con la ropa de cama de un hombre moreno llegado de Ceará, también internado en la Santa Casa, pero que sobrevivió. Desde entonces los casos se fueron expandiendo. Un oficio del ministro enviado al proveedor de la Santa Casa, participándole que la situación parecía estar agravándose, nos muestra la angustia de las autoridades, subrayada con el detalle de la hora: «Acabo de recibir (alrededor de la una de la mañana) un oficio de la Comisión Central de Salud

8. Era el principal hospital de la ciudad, administrado por la hermandad de la Misericordia.

9. Beltrão, Jane. Cólera, o flagelo da Belém do Grão-Pará. Belém: Editora UFPA; 2004, en especial el capítulo 2. David, Onildo Reis. O inimigo invisível: epidemia na Bahia no século XIX. Salvador: Editora da Universidade Federal da Bahia e Sarah Letras; 1996. Diniz, Ariosvaldo. As artes de curar nos tempos do cólera, Recife, 1856. In: Chalhoub, Sidney et al., orgs. Artes e ofícios de curar no Brasil. Campinas: Editora UNICAMP; 2009, p. 355-385.

10. Oficio de 13 de Jul de 1855. Localizado en: Archivo de la Santa Casa de la Misericordia de Rio de Janeiro (de aqui en adelante ASCMRJ), Registro de ofícios (1854-1856) [recibidos por el administrador]. 
Pública dando parte de haberse producido ayer por la tarde tres casos más de cólera morbus en el Arsenal de la Guerra» ${ }^{11}$.

En este artículo vamos a analizar cómo estaba organizada la asistencia a la población de Río de Janeiro a mediados del siglo XIX. Para ello, es importante considerar que la asistencia sanitaria y el ejercicio de la medicina estaban pasando por transformaciones significativas durante este período. El propio espacio donde se daba la asistencia hospitalaria se estaba modificando.

Al centrar la atención en las primeras grandes epidemias del Ochocientos en la capital del Imperio brasileño se pretende comprender mejor las opciones de la población, médicos y Estado, en términos de atención sanitaria en ese momento crítico. Estudiaremos las relaciones entre los grupos involucrados en relación a la atención en salud, como autoridades gubernamentales y médicas, población afectada y médicos, y la dirección de la Santa Casa de Misericordia, responsable del hospital más importante de la ciudad.

Para este análisis utilizamos como fuentes la correspondencia entre el gobierno y la Santa Casa de Misericordia, los informes anuales de ésta, los oficios de la Junta Central de Higiene Pública y las revistas médicas de amplia circulación en las décadas de 1840 y 1850.

\section{El hospital de la Santa Casa y la atención sanitaria en Río de Janeiro en el siglo XIX}

En Brasil, a lo largo del siglo XIX, la mayoría de la población buscaba tratar sus enfermedades en el hogar, a menudo recurriendo a terapeutas no reconocidos oficialmente. En las ciudades grandes, los pacientes que podían pagar los gastos de un internamiento, podían recurrir a tratamientos en clínicas. También había pequeños hospitales de hermandades destinados principalmente a atender a sus miembros.

11. Oficio de 1 Ago 1855. Localizado en: ASCMRJ, Ofícios Santa Casa de la Misericordia (18501857). Los médicos que la componían eran Paula Cândido, como presidente, Félix Martins, Pereira Reis, Pereira Rego y Pacheco da Silva, todos de la Junta, además de Góes Siqueira, Valladão Pimentel, Haddock Lobo, Lassance Cunha y Gonçalves Fontes. Jornal do Comercio (en adelante JC). 10 Sep 1855. 
El hospital de la Hermandad de la Misericordia se destacaba por estar abierto a personas de cualquier grupo social, condición jurídica (esclavo, liberto o libre), edad y color. La importancia del hospital residía también en la proximidad con la Facultad de Medicina. La presencia de profesores y alumnos ayudó a modificar la actividad cotidiana del hospital, al mismo tiempo que modificó la formación médica, pues los estudiantes tenían acceso a personas que padecían diversas enfermedades y a la farmacia, e incluso algunas clases teóricas fueron dictadas allí durante un determinado período.

Gran parte de los ingresos de la Santa Casa provenía de donantes cristianos que, además de ejercer la caridad y cuidar de la salvación de sus almas, adquirían prestigio social por pertenecer a la Hermandad. Entre los donantes, había personas vinculadas de alguna forma al gobierno, inclusive el propio emperador y ministros, lo que proporcionaba un constante diálogo con la administración de la Santa Casa. Esta proximidad entre la administración del hospital y el gobierno se reforzaba en parte por subvenciones concedidas a la Misericordia por medio de derechos sobre loterías y por el pago por la atención a grupos específicos como africanos nuevos, marineros y pacientes afectados por epidemias, siendo cobrado por la Santa Casa en diversas situaciones ${ }^{12}$.

En contrapartida, al designar a la Misericordia como local de clases prácticas y teóricas de la Academia Médico-Quirúrgica (1813-1832)/Facultad de Medicina de Río de Janeiro (después 1832), el Estado interfería directamente en su vida cotidiana, y ello generaba conflictos, por ejemplo al proponer que determinados médicos fuesen empleados en el hospital o al definir los castigos que podían ser aplicados a sus empleados. Interfería también cuando encaminaba algunos grupos de inmigrantes recién llegados para que fuesen atendidos en el hospital.

12. Algunos trabajos sobre la caridad y la Santa Casa de la Misericordia en diversos contextos, son Melo, Mariana de. Santa Casa da Misericórdia do Rio de Janeiro - assistencialismo, solidariedade e poder (1780-1822). Rio de Janeiro: Pontifícia Universidade Católica do Rio de Janeiro; 1997. Mesgravis, Laima. A Santa Casa de Misericórdia de São Paulo (1599?-1884). São Paulo: Conselho Estadual de Cultura; 1976. Abreu, Laurinda. Memórias da alma e do corpo - a Misericórdia de Setúbal na Modernidade. Viseu: Palimage Editores; 1999. Sá, Isabel dos Guimarães. Quando o rico se faz pobre: misericórdias, caridade e poder no império português, 1500-1800. Lisboa: Comissão Nacional para as comemorações dos descobrimentos portugueses; 1997. Russel-Wood, Andrew J. R. Fidalgos e filantropos: a Santa Casa da Misericórdia da Bahia, 1550-1755. Brasília: Editora da Universidade de Brasília; 1981. Sin embargo, existe una laguna historiográfica sobre los hospitales de las Misericordias en Brasil. 
La relación entre el Estado y el hospital de la Misericordia, sin embargo, se fue modificando a lo largo del tiempo, dependiendo de los grupos que tenían el poder en cada espacio, de la presencia de los médicos académicos en las enfermerías y de las amenazas concretas de epidemias. Tras la independencia política de Portugal (1822), la relación entre la Santa Casa y la Corona se puso de manifiesto en la elección de algunos administradores ${ }^{13}$.

Durante el periodo imperial (1822-1889), existieron 28 administradores, todos los cuales estaban en posesión de un título de nobleza ${ }^{14}$. La proveeduría de José Clemente Pereira (1838-1854) se diferenció de las anteriores por su duración y por la presencia de Pereira en los espacios de la Santa Casa y del gobierno. El proveedor, además de haber sido Consejero del Estado, fue diputado y senador, y ocupó las carteras del Imperio, Guerra, Justicia y Hacienda ${ }^{15}$. Al parecer, con él se inauguró un nuevo perfil de proveedor, pues muchos de los que le sucedieron también ejercieron esos mismos $\operatorname{cargos}^{16}$. Es interesante notar que en Río de Janeiro, capital del Imperio brasileño, la esfera en la que se movían los miembros de la hermandad y administradores estaba bastante más próxima a la Corona que en el caso de Casas de Misericordias de otras ciudades. Durante la administración de Clemente Pereira prevaleció un buen entendimiento entre la Santa Casa y la Corona. Esta íntima relación con el gobierno parece que benefició a la Misericordia, pues durante su administración logró mantener en equi-

13. La Santa Casa era dirigida por un administrador principal («provedor») y por una comisión («mesa»). Cuando utilizo el término administrador, me refiero al administrador principal.

14. Tres administradores estuvieron en el cargo en períodos diferentes, sumando 31 administraciones, si contamos los ejercicios consecutivos como una administración.

15. La mayor parte de los 16 administradores, que asumieron antes de 1838, lo hicieron por el mandato de un año. Pocos (tres) fueron reconducidos por más uno o dos mandatos, así como un número reducido ocupó cargos políticos (cinco) en el ejecutivo (1 ministro) o en el legislativo (como concejales, diputados o senadores). Entre las 15 proveedurías que existieron entre 1838 y 1889, seis fueron ejercidas por menos de un año para completar el periodo, como mandatos tapones, en general, a consecuencia del fallecimiento del administrador anterior. Incluso estos escogidos para quedarse pocos meses en el cargo también eran senadores, diputados, ministros y/o directores del Banco do Brasil.

16. Por ejemplo, los proveedores Miguel Calmon du Pin Almeida, Marques de Abrantes (1857-1865); Zacarias de Góes e Vasconcellos (1866-1877); José Ildefonso de Souza Ramos, Visconde de Jaguari (1879-1883); João Mauricio Wanderley, Barão de Cotegipe (1883-1889); y Honório Hermeto Carneiro Leão, Marques de Paraná (1855-1857). 
librio sus cuentas, inició y terminó la construcción de su nuevo hospital y consolidó un nuevo orden en su cotidiano ${ }^{17}$.

El nuevo hospital debería ser concebido de acuerdo con los conocimientos médicos de la época. Por lo tanto, se solicitó a la Academia Imperial de Medicina que trazara las «bases higiénicas para la construcción de un hospital». Entre las directrices generales, se aconsejaba que el hospital tuviera forma cuadrada, con uno de los ángulos apuntando hacia el este y el otro hacia el oeste, y que no hubiera árboles para no obstaculizar la libre circulación del aire y evitar la humedad de las paredes. Se daban, también, recomendaciones sobre las características del suelo, el techo y las tejas. Las salas de enfermos deberían estar aisladas unas de otras, contar con salas especiales para enfermedades contagiosas y para los recién operados, pues la convivencia y el testimonio del dolor del otro causaría desanimo profundo a quienes estaban a la espera de ser operados. En el terreno colindante, se podría construir una sala de autopsias y otra de lecciones clínicas, además de un jardín con una parte para el recreo de los convalecientes y otra para la distracción de los locos curables.

Detallando el proyecto, en un «informe descriptivo de la planta del nuevo hospital de la Santa Casa de la Misericordia de la ciudad de Río de Janeiro», la comisión especificaba el tamaño y cantidad de ventanas y puertas. Las ventanas deberían ser suficientemente altas, llegando cerca del techo, para facilitar la salida de las «emanaciones deletéreas y gases viciados, [que] por ser en general más leves que el aire», se concentrarían en la parte superior de una habitación. Como había gases más pesados, que también podrían comprometer la calidad del aire, era necesaria una pequeña apertura debajo de cada ventana. Se establecía asimismo que cada una de las 20 salas de enfermos tuviese capacidad para 32 lechos, sumando un total de 640. Y los lechos, de cuatro palmas de ancho, estarían separados por tres palmas de un lado y seis por otro ${ }^{18}$. El cambio de grandes salas comunes de enfermos, donde se colocaban más de cien personas, por pequeños espacios separados se justificaba, en general, por la necesidad de preservar a los enfermos de los miasmas producidos por otros en peor

17. Abreu, Laurinda, ed. Igreja, caridade e assistência na Península Ibérica (sécs.XVI-XVIII): estratégias de intervenção social num mundo em transformação. Lisboa: Edições Colibri/CIDEHUS/EU; 2004.

18. Novo Hospital da Santa Casa - 1840. Localizado en: ASCMRJ. 
estado. En el primer pavimento, fueron abiertas ocho de clínica interna y, en el segundo, seis de clínica externa.

De esa forma, las dos enfermerías existentes al comienzo del siglo XIX se dividieron a mediados de siglo en catorce, más dieciséis habitaciones de un lecho y dieciséis, de dos ${ }^{19}$. De un médico, un cirujano, un boticario y algunos enfermeros y sirvientes, a comienzos del siglo, la Santa Casa pasó a administrar, en la década de 1850, el trabajo de ocho médicos y sus respectivos sustitutos, un boticario y sus diez ayudantes, veintisiete pensionistas, veintiséis enfermeros/as, veintiocho sirvientes y dieciocho hermanas de caridad, a pesar de haber variado bastante durante los periodos de epidemia. La presencia más continuada de los médicos en el día a día del hospital trajo una organización más basada en el conocimiento médico y una unificación del lenguaje. La unificación terminológica fue coronada en 1852 con el catálogo de nosografía que identificaba algo más de 300 enfermedades, incluyendo sus variaciones.

Después de la inauguración del nuevo hospital, las estadísticas demuestran, a ojos del cuerpo médico, la eficiencia de la aplicación de las reglas de higiene. En 1853, la mortalidad en las enfermerías del viejo hospital era de $30,8 \%$, contrastando con los $8,9 \%$ encontrados en el nuevo ${ }^{20}$. Hasta finales de la década de 1850, la mortalidad promedio de los hospitales nuevo y viejo variaba alrededor de un $16 \%$, cuando se sacaba del número de muertos aquellos que habían fallecido las 24 horas después de haber sido internados. Al comentar la tasa de mortalidad en el hospital, los administradores discutían las cifras de muertos en las primeras 24 horas, 48 horas y 72 horas después del internamiento. Consideraban que, en esos casos, la culpa de la muerte sería de los propios enfermos que buscaban atención hospitalaria cuando ya estaban muy debilitados y la medicina académica no podría ayudar más. De hecho, podemos entender esa situación como una estrategia usada por familiares de enfermos o señores de esclavos que llegaban agonizando para tener acceso gratuitamente a los servicios de entierro de la propia Santa Casa de la Misericordia.

La importancia de la estadística en la afirmación del hospital bajo el comando predominante de los médicos académicos puede comprobarse con

19. En otro documento, se hace referencia a 16 salas para enfermos de 1 y 2 lechos.

20. Mapa de los enfermos que se trataron en el Hospital de la Santa Casa de la Misericordia del 1 de julio de 1852 hasta el 30 de junio de 1853. Localizado en: AN, IS 3 5. En los informes, los años se referían al período de julio del año anterior a junio del año referido. 
la institución del cargo de «médico director de estadística civil y médica del hospital y salas públicas de enfermos públicas». Independientemente de la forma como esos datos se produjeron, ayudaron a consolidar el poder de los médicos y contribuyeron a iniciar otro cambio en lo relativo al tipo de enfermo considerado adecuado para ser internado: un enfermo curable por la medicina académica ${ }^{21}$.

La administración buscaba estimular la búsqueda de personas que pudieran pagar por los servicios. Podrían elegir médico, tendrían más comodidad, mejores ropas de cama y habitación individual o compartida de a dos. Eso ayudaría a equilibrar el presupuesto de la Misericordia. En el nuevo reglamento había un artículo que se refería especialmente a los enfermos, buscando la expansión de los grupos sociales que podían ser atendidos en la Misericordia. Estaban divididos en tres clases: «1 ${ }^{\mathrm{a}}$ Clase: pieza para un enfermo solamente, con tratamiento especial, $2 \$ 000 ; 2^{a}$ Clase: pieza para dos enfermos, con tratamiento igual a los de la $1^{\text {a }}$ Clase, $1 \$ 600 ; 3^{\text {a }}$ Clase: en salas para enfermos con 32 lechos, siendo persona libre $1 \$ 000$, si fuera esclavo 800 réis» 22.

De hecho, después de la inauguración del nuevo hospital, en las décadas de 1830 y 1840, el número de enfermos tratados por su cuenta aumentó proporcionalmente en cerca de $10 \%$ y, al comienzo de la década de 1850, en $17 \%$. Por ejemplo, en el año administrativo de 1835-1836, el ingreso habitual sumó poco más de $103 \operatorname{contos}^{23}$, de los cuales cerca de 10 provenían de los pagos por tratamientos. En el año de 1846-1847, de manera aproximada, esos valores eran: 188 contos y 15 contos, respectivamente. Ya en 1854-1855, el ingreso habitual era de 263 contos y el pago por curaciones sumaba 44 contos. También llama la atención en los estados contables de ingresos y gastos, la importancia de los rendimientos de los «edificios urbanos» en el presupuesto, llegando a componer el $61 \%$ de los ingresos habituales. En el año administrativo de 1835-1836 alcanzaron 54 contos (52\%); en 1846-1847, 103 contos (55\%); y en 1854-1855, 160 contos $(61 \%)^{24}$.

21. Pimenta, Tânia Salgado. O Exercício das Artes de Curar no Rio de Janeiro (1828 a 1855). Campinas: Universidade Estadual de Campinas; 2003.

22. Regimento provisório do Novo Hospital da Santa Casa da Misericórdia do Rio de Janeiro. Rio de Janeiro: Typographia Nacional; 1852. Arquivo da SCMRJ, lata 10.

23. Referirse a 1 conto era lo mismo que decir 1 milion de réis, la moneda brasileña en la época. A mediados del siglo XIX, 1 pence inglés equivalía en media a cerca de 28 réis. Mattoso, Kátia de Queirós. Ser Escravo no Brasil. São Paulo: Ed. Brasiliense; 1990.

24. Informes de la Santa Casa de la Misericordia de Rio de Janeiro. Localizados en: AN, IS 3 3-5. 
Algunos de los edificios habían sido adquiridos por la Santa Casa, pero la mayoría procedían de donaciones a la hermandad a través de testamentos. Esas donaciones fueron disminuyendo con el tiempo y se fueron dedicando a gastos extraordinarios, como obras del nuevo hospital y del hospicio Pedro II, pero a pesar de ello constituían la base del patrimonio de la Misericordia. En los informes de diversos años, el crecimiento de los ingresos era explicado en función del reajuste de los alquileres y de una mayor fiscalización sobre los pagos ${ }^{25}$.

\section{La creciente demanda asistencial en el Hospital de la Santa Casa}

Año tras año, los proveedores de la Misericordia advertían el crecimiento de la demanda de servicios hospitalarios. La Hermandad de la Misericordia consideraba que su hospital estaba dirigido para el tratamiento de la pobreza, sin embargo, durante la primera mitad del siglo XIX, se quejaba con frecuencia de la flexibilidad con que se aceptaban enfermos como pobres y esclavos de señores que se decían pobres. Por este motivo, resultaba imprescindible redefinir lo que era ser pobre para facilitar la fiscalización.

En 1827, el artículo $16^{\circ}$ del estatuto del hospital establecía que: «Serán admitidos como pobres: $1 .^{\circ}$ Aquellos que presentaren comprobación de su respectivo Párroco en que atestigüe su pobreza. $2 .^{\circ}$ Los que hicieren constar su pobreza por información de palabra o por escrita de persona reconocida. $3^{\circ}$ Los marineros, presentando en el acto de su entrada, documento firmado por el propietario, capitán, o capataz de la embarcación a la cual pertenecen. $4^{\circ}$ Los esclavos de aquellos señores, que mostrasen no poseer más que dos esclavos». En el reglamento del nuevo hospital de 1852, en lo referente a la posesión de esclavos, se alteraba la definición de pobre como aquel que tuviera solo un esclavo.

El aumento de ingresos no era únicamente retórica de la administración para conseguir más privilegios del gobierno, pues el crecimiento del número de enfermos fue progresivo en los primeros años de la década de 1850. Mientras que en la década de 1830 y mediados de los años 1840, no se encontraban a diario en el hospital más que 400 personas internadas, menos de 5.000 por año, a mediados de la década de 1850, había entre 750

25. Informes de la Santa Casa de la Misericordia do Rio de Janeiro. Localizados en: AN, IS 33. 
a 850 personas por día, que sumaron más de 8.000 durante el año 1856 conforme vemos en la gráfica abajo ${ }^{26}$.

Gráfica 1: Número de enfermos internados en el Hospital de la SCMRJ (1853 a 1860)

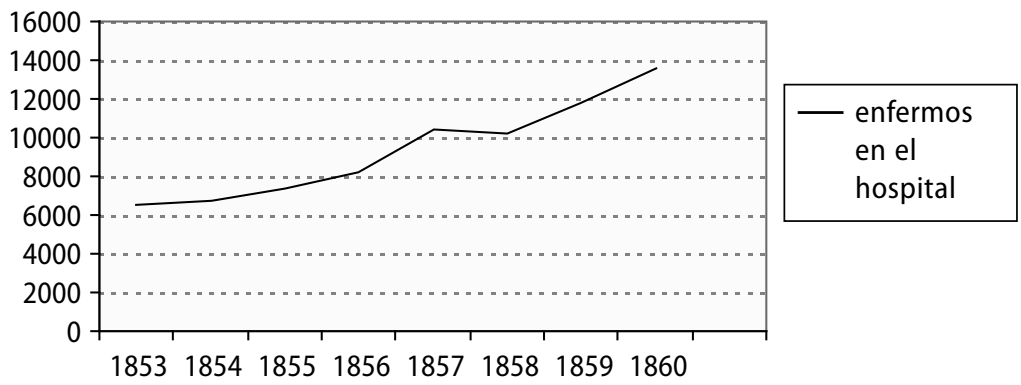

Fuente: Informes de la Santa Casa de la Misericordia de Río de Janeiro. AN, IS 3 5-6.

La ciudad de Río de Janeiro tuvo, durante la primera mitad del siglo XIX, un crecimiento significativo en términos económicos y de población. En 1821, la ciudad tenía 86.323 habitantes, de los cuales 40.376 eran esclavos; en 1849, había 205.906 moradores, siendo 78.855 esclavos. Una vez finalizado el tráfico atlántico de esclavos, en 1850, el número de esclavos declinó constantemente hasta la abolición en $1888^{27}$. Sin embargo, la grafica 2 muestra que el número de esclavos internados no acompaño la variación de la población esclava en la ciudad. Consideramos, por tanto, que los esclavos enfermos eran tratados por otros tipos de terapeutas y en un ambiente diferente del hospital.

Por otro lado, en la misma gráfica resulta evidente el crecimiento de los internamientos en el hospital de personas libres extranjeras, lo que se relaciona con el aumento de la inmigración europea. Los emigrantes llegaban a Río para dedicarse a diversas actividades y muchos de los que circulaban por la ciudad estaban de paso, como parte de la tripulación de

26. Informes de la Santa Casa de la Misericordia de Rio de Janeiro. Localizados en: AN, IS 3 3-5.

27. Karasch, Mary. A vida dos escravos no Rio de Janeiro (1808-1850). São Paulo: Companhia das Letras; 2000, p. 107-110. 
algún buque mercante o de camino a otra provincia. Constituían un gran número de personas sin lazos sociales establecidos que pudiesen apoyarlos y orientarlos en caso de enfermedad sin más opciones que el tratamiento en el hospital de caridad.

Gráfica 2: Internamiento en el hospital de la SCMRJ según la condición jurídica y la nacionalidad (1853 a 1857)

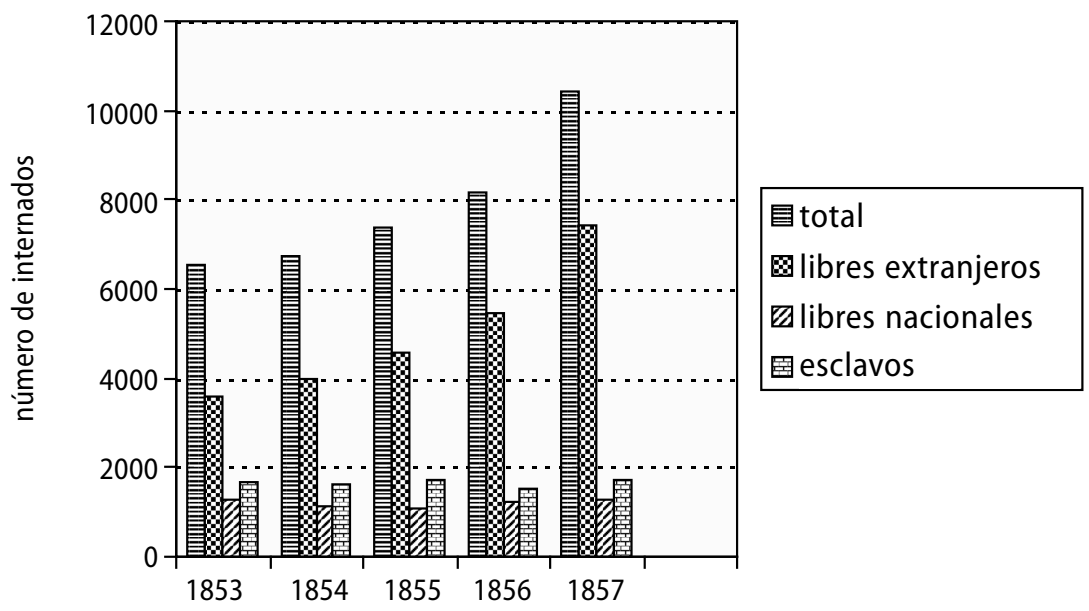

Fuente: Informes de la Santa Casa de la Misericordia de Río de Janeiro. AN, IS 3 5-6.

Se debe resaltar que estos números presentados en la grafica 2 no incluyen personas con «dolencias consideradas contagiosas para los cuales existe sala de enfermos especial». El informe referente al año de 1856 identificaba claramente que el aumento sería debido al progresivo aumento de la población extranjera, «puesto que, habiendo llegado a haber diariamente 878 enfermos, este número se componía de 118 personas nacionales, 600 extranjeros y 160 esclavos. Por regla general, el número de extranjeros es siempre el quíntuplo, si no séxtuplo, de los nacionales y el mayor actualmente» ${ }^{28}$.

28. Es importante señalar que no se encontraron los libros de entrada y salida de los enfermos del hospital de la SCMRJ en el AN y en el ASCMRJ. 
De hecho, el aumento del número de enfermos internados fue acompañado por el número de extranjeros internados, mientras el número de libres nacionales y el de esclavos permanecieron estables ${ }^{29}$. Por un lado, eso puede indicar la posibilidad de los extranjeros, entendidos como europeos, de ser más vulnerables a ciertas enfermedades con las cuales no habían tenido contacto anterior ${ }^{30}$. Puede indicar también la probable preferencia por otros modos de curar y la existencia de redes de solidaridad, de amparo, que evitarían, en muchas ocasiones, la terapéutica hospitalaria académica ${ }^{31}$.

En diversos momentos se observa un interés explícito de la Corona en proporcionar atención médica a grupos extranjeros. A través de oficios se ordenaba, por ejemplo, que la Misericordia atendiese a colonos irlandeses, españoles y alemanes y a los propios africanos libres ${ }^{32}$. Los costos del tratamiento de los inmigrantes enfermos eran cobrados, posteriormente, al gobierno que con frecuencia postergaba el pago a la Santa Casa.

\section{En tiempos de epidemias}

Teniendo en cuenta las transformaciones por las cuales estaban pasando el ejercicio de las artes de curar y la propia organización del principal hospital de la ciudad en la época, podemos entender mejor el impacto de la epidemia en la limitada estructura de atención a la salud y las soluciones propuestas para enfrentarlas.

Con la epidemia de fiebre amarilla de 1849-1850, se inició un intento de organización de los servicios de salud, como fue la creación de la Junta

29. Los informes referentes a los años de 1853 a 1857 presentan los números de enfermos internos clasificados en las categorías «libres nacionales», «libres extranjeros» y «esclavos» (en algunos años, separados en «esclavos nacionales» y «esclavos extranjeros»), además de la subdivisión por género.

30. Kiple, Kenneth. The Caribbean slave - a biological history. Cambridge: Cambridge University Press; 2002.

31. Según algunos estudios de la historiografía de la esclavitud, Engeman, Carlos. Vida cativa: condições materiais de vida nos grandes plantéis do sudeste brasileiro do século XIX. In: Fragoso, João et al., orgs. Nas rotas do Império. Vitória-Lisboa: IICT-EDUFES; 2006, p. 437. Moreira, Paulo. Os cativos e os homens de bem - experiências negras no espaço urbano. Porto Alegre: EST Edições; 2003.

32. Africanos que llegaron a Brasil a través del tráfico de esclavos después de su prohibición en 1831. Esas personas quedaban bajo custodia del Estado imperial cuando los buques negreros eran aprehendidos. 
de Higiene Pública, y una reestructuración de la atención sanitaria ofrecida a la población, además de acciones directas sobre la Santa Casa.

En este sentido, en 1851, la Santa Casa recibió una concesión para el establecimiento y mantenimiento de los cementerios públicos en los suburbios de Río de Janeiro por cincuenta años con el gravamen de mantener tres salas permanentes de enfermos para atender a los pobres. Esta obligación, sin embargo, fue negociada por algún tiempo, ya que en 1853 los sitios donde estarían ubicados todavía se estaban definiendo. En 1854, la hermandad fue relevada del mantenimiento de las salas comunes de enfermos, debido a las deudas contraídas para adquirir los terrenos para el cementerio, con la salvedad de que en tiempos de epidemias debería abrir tres ${ }^{33}$.

La primera gran epidemia de fiebre amarilla en el siglo XIX fue motivo de gran preocupación para el gobierno que pretendía estimular la inmigración de trabajadores europeos, precisamente los más afectados por la enfermedad $^{34}$. En esa época, la Santa Casa administró seis salas de enfermos externas a su hospital, que estaban en la isla de Bom Jesús, en el Hospicio do Livramento (en Playa Formosa, situado en la calle Saco y en la calle de la Misericordia). La Misericordia fue elegida por el gobierno ya que estaba preparada para instalar y administrar salas comunes de enfermos donde el gobierno considerara importante. La mayoría de las salas comunes de enfermos funcionó por algunos meses, pero la de la Isla de Bom Jesús tuvo que ser cerrada debido al «clamor general» de la población, horrorizada con el aislamiento de esta sala donde la mortalidad sería altísima ${ }^{35}$.

A cargo del servicio médico, de la contratación de médicos, enfermeros y ayudantes, del alquiler de la casa y de la compra de medicamentos, alimentos y suministros de oficina, la Santa Casa llevaba las cuentas de forma muy detallada para facilitar que el importe fuera reembolsado por el Tesoro Nacional.

La expectativa de la llegada del cólera desde hacía algunas décadas, había permitido cierta acumulación de conocimiento. La experiencia adquirida en la época de la fiebre amarilla, cuando la atención se organizó en las parroquias con comisiones médicas, formadas por médicos residentes en

33. Decreto n. 583 de 5 Sep 1850; Decreto n. ${ }^{\circ} 43$ de 18 Oct 1851; Decreto n. ${ }^{\circ} 75$ de 2 Sep 1854; y AN, IS 34 e 5 .

34. Chalhoub, Sidney, Cidade febril - cortiços e epidemias na corte imperial. São Paulo: Companhia das Letras; 1996.

35. AN, IS 34 . 
el lugar, sirvió también en 1855 para hacer frente al nuevo flagelo. Cuando los primeros síntomas de la enfermedad fueron reconocidos, la ciudad se dividió rápidamente en comisiones médicas, una para cada parroquia, que deberían contar con (al menos) un puesto médico. Los domicilios de los médicos se convirtieron en una referencia para la población. Estas informaciones y muchas otras fueron publicadas constantemente en los periódicos de mayor circulación en la ciudad.

A fines de septiembre de ese año, cuando la epidemia estaba próxima a alcanzar su auge, se podía leer en las publicaciones de la ciudad:

«Por orden del Ilmo. Sr. Dr. Luiz da Cunha Feijó, presidente de la comisión parroquial sanitaria de la parroquia de la Candelaria, hago público que se encuentra funcionando el puesto médico de esta parroquia, establecido en la calle Sabão ${ }^{\circ}$. 68, donde serán administrados a los individuos atacados por la enfermedad reinante los necesarios socorros en la forma de las respectivas instrucciones. Igualmente, hago público que la parroquia se encuentra dividida, para facilidad del servicio, en seis distritos médicos de la forma siguiente, comprendiendo:

El $1^{\circ}$ distrito. - La calle Violas a partir de la calle Ourives hasta la playa Mineiros; se encuentra a cargo del Sr. Dr. Joaquim Ferreira dos Santos Lima, habitante en calle Candelaria ${ }^{\circ}$. 40 .

El $2^{\circ}$ distrito. - La calle S. Pedro, y las transversales situadas entre ésta y Violas, a cargo del Sr. Dr. Antonio Pereira Leitão, calle Ourives nº 114.

El $3^{\circ}$ distrito. - La calle Sabão, y las transversales entre ésta y S. Pedro; a cargo del Sr. Dr. Francisco de Paula Costa, explanada Capim nº 75.

El $4^{\circ}$ distrito. - Las calles Alfândega y Hospício, y las transversales entre Sabão y esta última; a cargo del Sr. Dr. Francisco Joaquim de Souza Motta, calle Rosário $\mathrm{n}^{\circ} .60$.

El $5^{\circ}$ distrito. - Las calles Rosário de Ouvidor y las transversales, entre Hospicio y Ouvidor, a cargo del Sr. Dr. José Maria de Andrade, calle Quitanda $\mathrm{n}^{\circ} .85$.

El $6^{\circ}$ distrito. - Las calles Cano, Beco dos Barbeiros, Largo do Paço, Arco do Teles, Praça do Mercado y las transversales entre Ouvidor y Cano, a cargo del Sr. Dr. José Joaquim da Silveira, calle Misericórdia nº. 24». ${ }^{36}$

Del mismo modo, la asistencia a la población de otras parroquias urbanas fue organizada a medida que la enfermedad iba avanzando en la ciudad. Así, informaciones semejantes eran publicadas en esos días de 
temor sobre las parroquias de Gloria, São José, Sacramento, Candelaria, Santana, Engenho Velho.

Médicos y boticarios también divulgaban sus servicios gratuitos a la pobreza, así como las personas que donaban dinero o alimentos y ropas tenían sus nombres anunciados. Ciertamente, esas actitudes estarían motivadas por sentimientos de compasión y temor de propagación de la enfermedad, pero también estaban impregnadas por la necesidad de demarcación del lugar que esos grupos más privilegiados ocupaban en la sociedad.

En épocas de epidemia, la Misericordia mantenía tres salas comunes de enfermos - São João Batista, Nossa Senhora da Saúde y Morundúa cambio de lo cual gozaba de la concesión de la administración de los cementerios públicos. En octubre de 1855, cuando el número de muertos alcanzó su pico, la Santa Casa se responsabilizó también de otras 10 salas comunes de enfermos fuera de su hospital. Grupos de médicos, también, abrieron otras salas, como las Enfermerías Homeopáticas de S. Sebastião y de S. Vicente de Paulo y el puesto homeopático en calle Direita 96.

Durante esos años de epidemias, la homeopatía gozaba de bastante popularidad debido, en parte, a la insatisfacción de una parte de la población con los tratamientos propuestos por muchos médicos alopáticos como sangrías, flebotomías, sudoración, eméticos y purgantes. El tratamiento homeopático sería más barato y, sobre todo, tendría más afinidad con las concepciones de la enfermedad y la cura de ciertos grupos sociales por llevar en consideración elementos no físicos en su doctrina. Se entiende, por tanto, una confluencia entre la preferencia de los esclavos, libertos y libres pobres por ese sistema terapéutico, según ha señalado Angela Porto ${ }^{37}$, y el hecho de que éstos constituyeran parte significativa de los afectados por el cólera. Entre el 18 de julio 1855 y junio 1856 fueron enterradas 4.922 personas, siendo 2.538 esclavos, o sea, el $52 \%$ de los muertos eran esclavos. Las estadísticas oficiales no hacen distinción entre libertos y nacidos libres, lo que nos permite suponer que la proporción de descendientes de africanos muertos por el cólera habría sido mayor ${ }^{38}$.

37. Porto, Ângela. A assistência médica aos escravos no Rio de Janeiro: o tratamento homeopático. Papéis avulsos. Fundação Casa de Rui Barbosa. 1988; 7. Pimenta, n. 21, 2003.

38. Mapa demostrativo del número de personas fallecidas sepultadas en los cementerios públicos (São Francisco Xavier e São João Batista), además de los cementerios particulares de Gamboa y de São Francisco de Paula. Localizado en: AN, IS3 5. 
Todo ello permite pensar que esta asistencia estaba dirigida a determinados grupos sociales. Parte de los gastos de la sala común homeopática Nossa Senhora da Conceição, administrada por la Santa Casa, era pagada por el gobierno, lo que demuestra el reconocimiento oficial a la popularidad de la homeopatía y la importancia de ofrecer este sistema a aquellos que buscaban su tratamiento, a pesar de los conflictos con la medicina alopática.

\section{Consideraciones finales}

El estudio del hospital de la Santa Casa de la Misericordia de Río de Janeiro durante la reorganización de la atención sanitaria ante el problema de las epidemias de fiebre amarilla y cólera desatadas a mediados del siglo XIX en Río de Janeiro pone de manifiesto la manera en que el Estado afrontó tal situación.

La proximidad de la Hermandad con los espacios del poder político se evidenciaba por el hecho de que muchos administradores de la Santa Casa ocupaban cargos políticos, legislativos y del ejecutivo y formaban parte de los círculos de confianza del emperador. Esta cercanía al poder, reforzada a partir de finales de la década de 1830, estaba relacionada con una situación más estable en las finanzas de la Santa Casa.

Aunque los costos directos relacionados con la salud pública no llegaban a un 5\% en la mayor parte del siglo XIX, con la excepción de momentos de epidemias como a mediados de la década de 1850 cuando sumaron más del $50 \%{ }^{39}$, el estado imperial subsidió la atención a la salud proporcionada por la Misericordia a través de la concesión de parte de sus ingresos con loterías e impuestos sobre los alimentos y vino, por ejemplo.

Aunque el hospital afrontara, año tras año, el desafío de dar cabida cada vez a un mayor número de pacientes, la principal dificultad parecía ser más estructural que financiera, de acuerdo con los informes de la institución. La oferta de asistencia hospitalaria, aunque se ampliara con la instalación de salas comunes públicas administradas por la Misericordia, no satisfacía la creciente demanda por este servicio.

39. Carvalho, José Murilo. Teatro de Sombras - a política imperial. Rio de Janeiro: IUPERJ; São Paulo: Vértice; 1988, especialmente p. 38. 
Ante la inminente amenaza de propagación de la fiebre amarilla y del cólera, las autoridades políticas y médicas siguieron utilizando la organización y estructura de la Hermandad de la Misericordia para manejar la situación y ayudar a la población afectada. En ese momento, el hospital de la Misericordia estaba organizado de acuerdo a las normas de la medicina académica que establecía reglas de comportamiento para todos los que circulaban en ese ambiente - desde los empleados y estudiantes de medicina hasta los visitantes y pacientes internados. Además de eso, instituyó nuevos procedimientos que garantizaban un control del proceso terapéutico, como los formularios de registro de dietas, medicamentos y seguimiento de la evolución de la enfermedad. Sin embargo, la edificación de las pequeñas salas comunes de enfermos, aprovechando los subsidios durante los períodos de epidemias, no fue suficiente para satisfacer la demanda de atención hospitalaria que aumentaba con el crecimiento de la ciudad.

El hospital de la Santa Casa de la Misericordia de Río de Janeiro se destacaba por ser el más grande de la ciudad durante el siglo XIX y estar abierto a cualquier grupo social. Este establecimiento fue testigo de importantes conquistas de la corporación médica en su lucha por el monopolio de las artes de curar, en gran parte debido a la proximidad con la Facultad de Medicina. De hecho, el crecimiento del número de enfermos tratados en el hospital es sorprendente. A pesar de la atención gratuita a pobres y de la reducción de la mortalidad hospitalaria, no podemos olvidar que la mayor parte de la asistencia continuaba en manos de sanadores populares con quienes compartían concepciones sobre salud y enfermedad. 
